\title{
Legitimação e "Universitarização" da Formação de Professores em Quebeque: surgimento de uma cultura da pesquisa ${ }^{1}$
}

\author{
THÉRÈSE HAMEL ${ }^{2}$ \\ Professora do Departamento de Fundamentos e de Práticas em Educação \\ da Faculdade de Educação da Universidade Laval \\ Therese.Hamel@fse.ulaval.ca \\ MARIE-JOSÉE LAROCQUE ${ }^{2}$ \\ Doutoranda da Faculdade de Educação da Universidade Laval \\ marie-josee.larocque@fse.ulaval.ca
}

\begin{abstract}
Resumo
Este artigo apresenta as descobertas preliminares de um estudo acerca do surgimento de uma cultura da pesquisa na área da formação de professores, focalizando especificamente o caso da Universidade Laval em Quebeque, Canadá. As autoras definem, primeiramente, alguns conceitos e discutem o período em que a formação de professores em escolas normais era muito rudimentar. Em seguida, concentram-se nos três tipos de instituições universitárias da época (escola normal superior, escolas universitárias de educação e faculdades de educação). Baseadas em certos indicadores, elas determinam como essa cultura da pesquisa se solidificou, entre outras coisas, na "universitarização" da formação de professores. Na segunda e última parte, Hamel e Larocque destacam alguns períodos de ruptura que revelam o aparecimento de uma nova organização de cultura da pesquisa
\end{abstract}

1 Este artigo é parte de um projeto mais abrangente chamado "Tradições e Transições na Educação de Professores: as experiências de Educação de Professores em Ontario, Quebeque e Saskatchewan, 1945-2002", patrocinado pelo Conselho de Pesquisa em Ciências Sociais e Humanas do Canadá (SSHRCC, sigla original em inglês). Também trabalham neste projeto Sandra Acker, Elizabeth Smyth e Jo-Ann Dillabough do Instituto Ontariano para Estudos em Educação na Universidade de Toronto (OISE/UT, em inglês), bem como Dianne Hallman da Universidade de Saskatchewan. Para uma panorama geral deste projeto, ver Acker (2000). Uma versão inglesa deste texto foi publicada na revista European Educational Research Journal, volume 1, number 1, 2002, p. 99-117. O título inglês era "Observations from Québec: the Emergence of a Research Culture in Education Through Legitimacy and Universitarisation, 1940-2000".

2 Thérèse Hamel é membro do Centro Interuniversitário de Estudos Quebequenses (CIEQ) e membro associado do Centro de Pesquisa Interuniversitária sobre a Formação e a Profissão Docente (CRIFPE). Marie-Josée Larocque é membro do Centro Interuniversitário de Estudos Quebequenses (CIEQ) e da Sociedade Canadense de História da Igreja Católica (SCHEC). 
anterior a 1955, emergindo verdadeiramente entre 1955 e 1969 e adquirindo legitimidade e uma posição dominante após 1969. Na conclusão as autoras destacam assuntos trazidos à tona devido a essa mudança na cultura institucional e argumentam que o surgimento de uma cultura da pesquisa em Quebeque e em outros lugares mudou as tradições e marcou as transições no desenvolvimento da educação e na formação de professores.

Palavras-chave: "universitarização", cultura da pesquisa, formação de professores.

\title{
Resumen
}

Este artículo muestra las descubiertas preliminares de un estudio sobre el aparecimiento de una cultura de investigación en el área de la formación de profesores, enfocando especificamente el caso de la Universidad Laval en Quebec, Canadá. Las autoras definen, en primer lugar, algunos conceptos y discuten el periodo en que la formación de los profesores de las escuelas normales era muy primitivo. En seguida, se concentran en los tres tipos de instituciones universitarias de la época (escuela normal superior, escuelas universitarias de educación y facultades de educación). Apoyadas en ciertos indicadores, ellas determinan como esa cultura de la investigación se consolidó, entre otras cosas, en la"universitarización" de la formación de los profesores. En la segunda y última parte del trabajo, Hamel y Laracque destacan algunos periodos de ruptura que revelan el aparecimiento de una nueva organización de la cultura de investigación anterior a 1955, surgiendo verdaderamente entre 1955 y 1969 y adquiriendo legitimidad y una posición dominante después de 1969. .En la conclusión las autoras destacan asuntos ventilados gracias a esa mudanza en la cultura institucional y argumentan que el aparecimiento de una cultura de investigación en QQuebec y en otros lugares cambió las tradiciones y marcó las transiciones en el desenvolvimiento de la educación y en la formación de los profesores.

Palabras-clave: "universitarización", cultura de la investigación, formación de profesores.

\begin{abstract}
This article sets out the preliminary findings of a study on the emergence of a research culture in the field of teacher training, focusing specifically on the case of Laval University in Quebec (Canada). The authors first define some concepts and discuss the period when teacher training in normal schools was highly rudimentary, then focus mainly on the three types of university institutions at the time (higher normal school, school of education and faculty of education). Based on a number of indicators, they determine how this research culture found a place for itself in, among other things, the universitarisation of teacher training. In the second part, the authors highlight some periods of disruption which reveal that a fledgling research culture appeared before 1955, truly emerging between 1955 and 1969, and gaining a dominant place and true legitimacy after 1969. The authors conclude by outlining the issues raised by this change in the institutional culture and argue that the emergence of a research culture in Quebec and elsewhere has changed the traditions and marked the transitions in the development of education and teacher training.
\end{abstract}

Key words: universitarisation, teacher training, research culture. 


\section{INTRODUÇÃO}

Este artigo mostra as descobertas de um estudo apresentado em um simpósio chamado "Fontes do surgimento e do recente desenvolvimento da pesquisa educacional como disciplina acadêmica: uma resposta às demandas de aumento da qualificação para as profissões educacionais", que ocorreu concomitantemente à Conferência Européia de Pesquisa Educacional (ECER) e à Associação de Pesquisa em Educação e Formação (AECSE, sigla em francês), realizado em setembro de 2001 em Lille (França). Nossa contribuição para esse simpósio trata, em particular, das tensões dinâmicas entre as duas principais abordagens para o desenvolvimento das faculdades de educação. Por um lado, desde a extinção das escolas normais, as faculdades de educação têm se especializado, entre outras coisas, na formação de professores e no ensino profissionalizante, tendo, portanto, grande importância na área de formação profissional de estudantes. Por outro lado, e ao mesmo tempo, esses profissionais são cada vez mais solicitados a fazer parte da cultura da pesquisa, que é altamente considerada pela instituição universitária. Neste artigo examinaremos, mais especificamente, um dos dois pólos deste problema dialético, ou seja, o papel da cultura da pesquisa e o seu desenvolvimento nas instituições especializadas na formação de professores, sobretudo as ligadas à Universidade Laval. Exploraremos as relações complexas entre a formação profissional oferecida na área de educação em universidades e as pressões exercidas nesta área pelo aumento da cultura da pesquisa em universidades ${ }^{3}$.

Sendo uma das muitas vias que podem ser exploradas, o presente estudo analisa esse assunto da perspectiva do surgimento de uma cultura da pesquisa nas faculdades de educação. Mais especificamente, usaremos uma aproximação histórico-social para compreender a legitimidade da "universitarização" da formação de professores em relação ao desenvolvimento da pesquisa. Focalizaremos especificamente o caso da Faculdade de Educação da Universidade Laval, um importante, porém mal documentado, fenômeno na história da formação de professores em Quebeque. Primeiramente, definiremos rapidamente alguns indicadores e a própria noção de cultura da pesquisa para então destacarmos algumas limitações deste estudo. Em segundo lugar, traçaremos os três períodos da formação de professores de Quebeque em suas principais instituições (escolas normais, escolas universitárias de educação e faculdades de

\footnotetext{
3 Ver Mathurin (1992). O autor estuda com maior precisão as transformações da Faculdade de Educação da Universidade de Montreal.
} 
educação). Em terceiro lugar, nos concentraremos principalmente na importância dos períodos-chave para o desenvolvimento da cultura da pesquisa nessas instituições. Por último, entraremos em questões que emergem da nossa visão panorâmica do desenvolvimento da cultura da pesquisa.

É necessário destacar primeiramente que, em Quebeque, o surgimento da pesquisa foi inicialmente examinado com base no desenvolvimento das chamadas disciplinas científicas ou de ciência pura. Chartrand et al. (1987) descreveram o desenvolvimento da pesquisa nas universidades de Quebeque, que foi, conforme citação abaixo, bastante diferente das universidades do Canadá Inglês:

"A pesquisa científica surgiu em primeiro lugar na Universidade McGill [uma universidade de lingua inglesa] em meados dos anos de 1890, enquanto na Universidade Laval e na Universidade de Montreal só se desenvolveu após 1920 em química e biologia, e depois de 1939 em física. Em matemática, a pesquisa tornou-se uma atividade imponente nos anos de 1960." (p. 381-382) [tradução]

Dirigindo-se a outra área de assuntos gerais, Roby (1996) examinou o desenvolvimento da pesquisa na Faculdade de Artes da Universidade Laval e mostrou que, embora incansáveis esforços tenham sido feitos nessa área, a pesquisa teve início somente na década de 70. Finalmente, Corbo (1994) afirmou que a pesquisa na Universidade de Quebeque em Montreal (UQAM) somente decolou da década de 80. Assim, serão exploradas neste estudo as seguintes questões: qual é a situação na área de educação? quando a cultura da pesquisa surge nas instituições responsáveis pela formação de professores, como, por exemplo, no caso da Universidade Laval?

A cultura da pesquisa é definida aqui como a cultura dentro de uma instituição que valoriza e facilita o desenvolvimento de novos conhecimentos, do básico à pesquisa aplicada. Isso significa um processo estruturado de exploração intelectual, intervenção e criação que conduz ao avanço do conhecimento. Na maioria das vezes, essas atividades podem ser disseminadas por meio de uma variedade de publicações tais como artigos, teses, livros e relatórios de pesquisa. Essa pesquisa pode ser feita individualmente ou por uma grande equipe com ou sem auxílio de bolsas. Na maioria dos casos, a cultura da pesquisa dentro de uma universidade parece estar ligada à obtenção de um grau de doutor. Além disso, o assunto da cultura da pesquisa não pode ser mencionado sem a devida colocação em relação a outras culturas institucionais, por exemplo a relacionada à

4 Ver os estudos de Calam (1994); Fullan (1996); Patterson (1979); Sheehan e Wilson (1994). 
prática profissional, que é mais diretamente ligada à prática de ensino. Embora não seja possível examinar isso no presente artigo, é importante situar a cultura da pesquisa em relação a outras culturas institucionais e ao ambiente que a envolve, ou seja, a política e a vontade de implementar uma cultura da pesquisa, exigências das autoridades (governo, Ministério da Educação, administração das instituições de formação de professores, da própria universidade, organismos que concedem bolsas) e assim por diante.

A fim de acompanhar o surgimento e as transformações dessa cultura da pesquisa, é importante estabelecer um número de indicadores que são resumidos a seguir ${ }^{5}$. O primeiro indicador diz respeito à inclusão da pesquisa no conjunto de atividades da instituição. Essa atividade é determinada e transmitida pelo discurso institucional por meio de calendários e outras publicações oficiais da universidade e da faculdade. Outra série de indicadores relaciona-se à qualificação dos professores em instituições educacionais, isto é, se elas têm ou não doutorado e se visam ou não a estudos de pós-graduação. Isto nos ajudará a entender o desenvolvimento e o crescimento de títulos de doutor entre professores. Essa duas maiores categorias de indicadores (atividade e qualificação dos professores) nos permitirão avaliar o surgimento da cultura da pesquisa. Examinaremos também, qualitativamente, algumas trajetórias individuais que ilustram como a pesquisa tem, através dos anos, se desenvolvido na Faculdade de Educação da Universidade Laval.

\section{OS PERÍODOS DA FORMAÇÃO DE PROFESSORES E DA CULTURA DA PESQUISA}

Para avaliar a importância dada à pesquisa nessa área e para simplificar a apresentação dos resultados, nos concentraremos primeiramente em três períodos cronológicos relacionados a diferentes grupos de instituições envolvidas na formação de profissionais em educação: 1) escolas normais; 2) escolas universitárias de educação; e 3) faculdades de educação. Embora essas instituições tenham sucedido uma à outra através dos anos, também se sobrepuseram como mostra a Figura 1.

\footnotetext{
5 Para os propósitos deste artigo, omitimos outros indicadores também importantes (desenvolvimento de programas de estudos avançados, mudança na clientela de estudantes, critério para contratação de professores, subsídios de pesquisa obtidos, e assim por diante).
} 
Figura 1: O surgimento de uma cultura da pesquisa em Educação

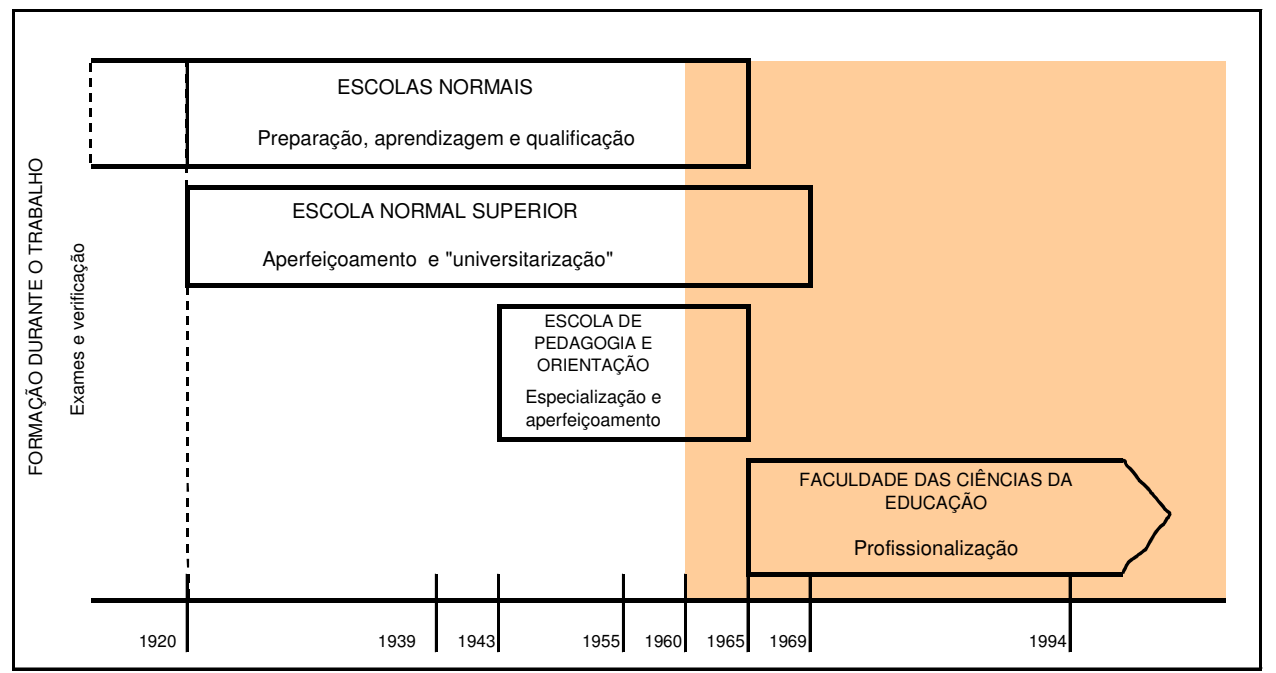

Fonte: Laboratório de geografia histórica, Centro Interuniversitário de Estudos Quebequenses.

Para entender o período estudado (1939-2002), precisamos retornar brevemente ao período que antecede a 1939. Naquele tempo, existiam dois modos concorrentes para se tornar um professor: freqüentar a escola normal ou passar em um exame da Banca de Examinadores para obter o certificado de professor. Durante todo esse período, a grande maioria dos futuros professores jamais passou por escolas normais e, conseqüentemente, nunca recebeu qualquer treinamento institucional (Hamel, 1995). A formação do professor era feita principalmente "durante o trabalho", com os portadores de certificados da Banca de Examinadores, por meio de uma prova que testava somente seus conhecimentos gerais. Além disso, as pessoas de ordens religiosas não eram obrigadas a realizar esse treinamento para professores, já que seu status era considerado um substituto para essa formação. O término das bancas de examinadores, em 1939, teve muitas conseqüências, uma das quais foi que, daquele momento em diante, a formação de professores dependeria exclusivamente das escolas normais, isto é, instituições especificamente destinadas a esse fim. Além disso, em vez de centralizar a formação de professores em poucas escolas normais, o governo decidiu expandir a rede de pequenas instituições existentes em Quebeque. Desse modo, a formação de professores se expandiu bastante de 1939 em diante. 


\section{O período das escolas normais (1939-1969)}

Até 1940, a legislação sobre formação de professores não havia mudado muito e as escolas normais tinham a seguinte missão: "preparar e instruir professores de escolas elementares na arte de ensinar"6. Então, no intervalo de 1940 a 1955, foi dada mais ênfase à parte do currículo relacionada à formação de professores do que à "cultura geral", principalmente porque surgiram os pré-requisitos. Entretanto, a reforma de 1953 e particularmente a criação do diploma Classe $A^{7}$ marcaram um ponto de mudança na maneira pela qual o currículo de conhecimento era organizado e estruturado. Foi durante esse período que o treinamento profissional ganhou verdadeira credibilidade e, a partir de então, desempenhou um papel mais importante nos programas. Ao introduzir psicologia, psicopedagogia e didática (Hamel 1991, 1995) as escolas normais tentaram melhorar a "parte profissional do currículo", ou seja, aquela que preparava os professores para ensinar.

De 1955 em diante, a elaboração das diretrizes de algumas das escolas normais foi especializar a formação de professores de escolas secundárias, aumentando assim seu nível de formação. Na metade da década de 1950, muitas mudanças foram feitas na organização das escolas normais estaduais. Uma delas foi a criação e o aumento da importância dessas escolas nos grandes centros da província. Essas novas instituições se especializaram na formação de professores de escolas secundárias, em contraste com as pequenas escolas normais dos pequenos centros da província, que eram mantidas por ordens religiosas e especializadas na formação de professores para escolas elementares (Hamel, 1995). A justificativa para a criação dessas novas instituições foi a necessidade da formação de professores, homens de preferência, para escolas secundárias. As escolas normais para meninas, que por séculos foram responsáveis pelo treinamento da maioria dos professores, foram substituídas pelas instituições maiores dos grandes centros da província.

$\mathrm{Na}$ verdade, nesse período, não houve sinais de uma cultura da pesquisa em escolas normais. Para aqueles que trabalhavam nessas instituições, o período das escolas normais foi caracterizado por um

\footnotetext{
6 Lei de 1851, Ato para prover o estabelecimento de uma escola normal e para melhor estimular a educação no Canadá meridional, 14-15 Vict, cap. XCVII, p. 39-40. Leis subseqüentes de 1877, 1925 e 1940 mantiveram a essência principal desta missão.

7 No nível do diploma Classe $\mathrm{A}$, os primeiros 2 anos de estudo são dedicados à consolidação de cultura geral. No terceiro ano, a ênfase é dada principalmente ao treinamento psicopedagógico e o quarto ano é dedicado principalmente à especialização e à metodologia das matérias a serem ensinadas.
} 
treinamento essencialmente centrado no preparo de professores para suas novas atividades. Embora a psicologia tenha sido introduzida na formação de professores, não havia nenhuma tipo de cultura de pesquisa institucionalizada, ao menos no que se refere às escolas normais que treinavam professores de escolas primárias. Isso, entretanto, não significava que as pessoas não realizavam pesquisa. Alguns indivíduos decidiam por conta própria completar o grau de mestre ou de doutor, escrever livros, envolver-se com pesquisa e, possivelmente, ter acesso a bolsas ou verba proveniente de ordens religiosas, a fim de realizar estudos no exterior ou em outras universidades. Porém, não havia nada que fosse institucionalmente organizado podendo ser descrito como uma tendência compulsória, que foi definida anteriormente como a presença de indicadores de pesquisa.

Antes que a formação de professores fosse oficialmente transferida para as universidades e que a cultura da pesquisa se tornasse tão importante quanto é atualmente, o primeiro passo foi aumentar o nível de pré-requisitos e de escolaridade. $\mathrm{O}$ fato de a formação universitária tornarse cada vez mais necessária, para futuros professores de educação primária e secundária, deu ímpeto a esse movimento. De fato, enquanto as escolas normais centralizadas funcionavam, outro movimento, paralelo e menos evidente, tomava forma: a longa marcha para as universidades e a organização das "escolas universitárias de educação". No item seguinte, examinaremos a questão da existência, ou não, de indícios de que a cultura da pesquisa se desenvolvia naquele momento.

\section{O período das escolas universitárias de educação}

A grande maioria das escolas normais mencionadas anteriormente foram instituições especializadas na formação de professores para escolas primárias. Entretanto, na década de 1920, as escolas universitárias de educação foram criadas: uma nova instituição cuja tarefa principal era treinar professores para escolas secundárias e normais. Elas também podiam oferecer cursos de aperfeiçoamento para aqueles que estavam na ativa. Gradualmente tornar-se-iam filiadas às universidades pois estas estavam interessadas no treinamento dessa categoria de professores. Examinaremos o caso específico de duas instituições que eram filiadas à Faculdade de Educação da Universidade Laval - a Escola Normal Superior (1920) e a Escola de Pedagogia e de Orientação (1943), que representaram dois modelos de formação de professores. 


\subsection{A Escola Normal Superior da Universidade Laval (1920-1970)}

Depois de muitos anos de esforços (Hamelin, 1995), a Escola Normal Superior (ENS) da Universidade Laval foi criada e, em 1920, tornou-se a primeira instituição da categoria na província de Quebeque. Essa instituição desempenhou um papel importante na formação de professores bem como na criação da Faculdade de Artes e no desenvolvimento da área científica. Sua proposta era treinar professores de escola secundária, que trabalhariam principalmente em colégios clássicos, e também professores da escola normal.

As diretrizes da missão refletem claramente o desejo de fornecer um nível universitário de educação a esses professores e abrir as portas das instituições anglo-canadenses e americanas aos franco-canadenses. Isso significava elevar o nível de qualificação dos professores. Seu desenvolvimento profissional era outro elemento importante na elaboração de diretrizes, particularmente em relação a cursos de verão. Mencionou-se que o treinamento durante o trabalho ajudaria a mostrar que a obrigação moral e educacional era atingida pela formação de professores na província e não no exterior. Voltado a leigos e religiosos, esse treinamento era destinado à formação de "professores católicos franceses". No entanto, embora a evolução na elaboração de diretrizes através dos tempos tenha revelado pequenas mudanças na orientação da instituição, de maneira suficientemente interessante, a instituição continuou a ser referência de um sólido treinamento básico de professores de escolas secundárias e na formação profissional necessária para sua carreira8 .

$O$ fato dessa instituição ter-se especializado na formação de professores de escolas secundárias parece significar que sua atividade educacional foi dividida em dois aspectos distintos: as disciplinas de ensino e a formação educacional. A visão da ENS pode ser entendida com base na dualidade dessas duas dimensões, $o$ fato de elas ocorrerem consecutivamente e a dificuldade de relação entre a ENS e as outras faculdades nos anos de 1960. Entretanto, durante um intenso período de reorientação do sistema de educação em Quebeque (1964-1965), a instituição ainda insistia na importância da formação de professores em suas disciplinas. A ENS também foi reformulada durante esse período. Essa reorientação significou que os cursos eram ministrados nas faculdades das universidades e que a ENS proporcionava o treinamento psicoeducacional necessário ao papel de educador. Essa divisão de tarefas entre as duas

8 No Calendário 1954-1955, por exemplo. 
entidades (disciplinas e pedagogia) dentro da universidade teria conseqüências no futuro, já que a ENS desejava manter a dualidade na organização do seu currículo.

Por um lado, existem muitos indicadores que sugerem que a formação de professores era o foco principal da ENS. Sua elaboração de diretrizes estabeleceu claramente a formação de professores como uma prioridade e, por fim, como a principal proposta da instituição. Outros indicadores incluíam a importância dada à prática em sala de aula, à existência de exercícios, à tendência de levar em consideração a personalidade do candidato e à presença de um diretor de treinamento de técnicas especializadas.

A questão que permanece é se a cultura da pesquisa emergiu ou não dessa instituição. A ENS ofereceu programas de mestrado e doutorado desde que foi criada, e isso certamente criou um forte movimento para a qualificação do nível universitário. ${ }^{9}$ A partir de 1945, começaram a ser vistos os primeiros sinais de uma preocupação em desenvolver uma cultura da pesquisa. Elementos relacionados a teses, publicações e critérios de elegibilidade para professores supervisionarem uma tese apareceram no calendário. Realmente, embora as diretrizes parecessem claramente orientadas para o treinamento de professores, a qualificação destes mostrou que a universidade aplicou um número de critérios, pelo menos não oficialmente, em contratar um determinado tipo de professor. Baseado em informações correntes e disponíveis, existe alguma indicação de uma cultura da pesquisa no critério de contratação de professores, embora esta via de investigação, em particular, ainda estivesse em um nível inicial. Por exemplo, dos três primeiros professores contratados, somente um tinha doutorado na França, porque era impossível encontrar candidatos com essas qualificações em Quebeque.

Comparada às escolas normais discutidas anteriormente, a ENS estava caminhando para a "universitarização" da formação de professores e estava aberta a alguns tipos de estudos graduados nessa área. Foi imperativo que os professores das escolas secundárias e normais melhorassem suas qualificações. Todavia, mesmo com o apoio financeiro ao programa de formação de professores dado pelo Governo de Quebeque ${ }^{10}$, parece que a pesquisa feita na ENS foi conduzida na perspectiva de examinar e estudar o assunto em profundidade em vez de

9 A existência, desde 1939, da Escola de pós-graduação também pode ter dado ímpeto para prosseguir nessa direção.

10 Lei para facilitar a formação universitária do corpo docente das escolas secundárias, das escolas normais e dos colégios clássicos assim como as pesquisas relativas ao ensino (8-9 Eliz II, ch. 26, 1961). 
ser apenas um primeiro passo para formar pesquisadores. Assim, enquanto havia alguns indicadores de uma nascente cultura da pesquisa, ainda existia o ritual ou prática de uma instituição principalmente dirigida ao preparo de professores. O objetivo do modelo de treinamento da ENS foi o de melhorar a qualificação dos professores, já que um número cada vez maior deles teve formação universitária, incluindo graus de mestre e de doutor. Apesar disso, não pode ser dito que a ENS, como instituição, mantinha seu foco na pesquisa, uma vez que isso não era seu objetivo educacional. Ainda assim, como veremos adiante, outra instituição, filiada à Universidade Laval e que desempenhou um papel importante na criação da Faculdade de Educação, estava caminhando para um modelo completamente diferente, altamente centrado na cultura da pesquisa.

\subsection{A Escola de Educação e Orientação Vocacional (1943-1965)}

A Escola de Pedagogia da Universidade Laval foi criada em 1943 e passou a chamar-se Escola de Pedagogia e Orientação (EPO) em 1947, quando se uniu ao Instituto de Orientação e ao Instituto de Psicoterapia. Tornou-se Escola de Ciências Pedagógicas e Psicológicas em 1964 antes de denominar-se Faculdade de Ciências da Educação em 1965.

A nosso ver, essa instituição foi a primeira a mostrar elementos tangíveis de uma cultura da pesquisa. Por exemplo, seu mandato expôs claramente o lugar da pesquisa no currículo do conhecimento. Diferentemente da ENS, essa instituição adotou um programa de pesquisa em seus primeiros calendários (1946-1947). Assim, enquanto a escola buscava melhorar o treinamento e o conhecimento educacional dos estudantes, bem como introduzir os métodos e tendências da educação contemporânea aos professores, a EPO queria, acima de tudo, estimular a pesquisa em educação entre os franco-canadenses por meio do treinamento dos educadores nos procedimentos da pesquisa científica em educação. Depois de alguns anos de reorganização, a escola dividiu seu programa de mestrado em três seções, uma das quais era especificamente voltada ao treinamento de pesquisadores. $O$ objetivo era compensar a falta de pesquisadores nessa área em Quebeque, já que as descobertas em pesquisa dos países de língua inglesa eram de difícil acesso em francês. O Comitê de Defesa da Pesquisa (DRB) e a Fundação Carnegie concederam bolsas para financiar essa parte da pesquisa.

A pesquisa conduzida pela EPO foi justificada pela natureza das diretrizes educacionais e vocacionais, que eram consideradas ciências experimentais. Além disso, a tarefa de uma parte deste departamento de pesquisa foi a de criar testes para selecionar estudantes em outras 
faculdades da universidade. Cursos introdutórios em pesquisa, estatística e metodologia eram dados, já que o grau de mestre exigia uma tese escrita. A instituição também tinha um laboratório de psicologia animal. De fato, a EPO já se considerava uma faculdade de alto nível educacional e tentava desenvolver um "espírito científico" nos estudantes. Assistentes de pesquisa foram, então, contratados pela escola, equipes de pesquisa começaram a se instalar e mais professores foram contratados. Por exemplo, em 1962, cinco professores da escola estavam trabalhando no Projeto Carnegie, para o qual dedicaram um terço do ano acadêmico e mais dois meses no verão. A noção de disponibilidade para atividades de pesquisa foi explicitamente notada nesta instituição.

Em suma, a EPO fazia, claramente, parte da cultura da pesquisa vinculada a outros mandatos, tais como supervisão escolar e ensino em escolas normais. Examinando o desenvolvimento da cultura da pesquisa, não podemos ignorar o fato de que dois membros centrais da instituição, Monsenhor Parent e Arthur Tremblay, seriam também os maiores idealizadores da reforma educacional em Quebeque. Eles também acreditavam que a pesquisa teria de desempenhar um papel de maior destaque na instituição antes de tornar-se a Faculdade de Educação da Universidade Laval.

\subsection{O período das Faculdade de Educação (1965-...)}

Por que uma instituição como a Escola de Pedagogia, em que o número de estudantes aumentava e os professores estavam sendo bem treinados - evidência de uma emergente cultura da pesquisa - abandona suas atividades e abre caminho para a Faculdade de Educação? Para entender esse movimento é importante saber que, na década de 1960, a EPO e a ENS estavam no centro da revolução que mudaria radicalmente a sociedade de Quebeque. Na verdade a "Revolução Silenciosa" transformou todos os segmentos sociais, incluindo o sistema educacional que foi organizado pela Comissão Parent, conforme a publicação do seu relatório em 1963. Tendo em mente as experiências americana e inglesa, o relatório Parent visou ao treinamento organizado de professores de acordo com um novo paradigma, isto é, a formação de professores estaria sob a responsabilidade da universidade.

Para realizar esse projeto, os comissários previram a criação de novas universidades, uma vez que acreditavam que as já existentes não poderiam treinar professores em toda a província de Quebeque, parcialmente devido à falta de infra-estrutura. Escolas normais, espalhadas por todo o território provincial, foram consideradas muito pequenas pela 
Comissão Parent e possuíam poucos estudantes para estarem aptas a fornecer um alto nível de treinamento. O nível dos diplomas (por exemplo, diploma Classe B) também foi considerado, por alguns críticos, como muito baixo para o preparo de bons professores. $\mathrm{O}$ desenvolvimento profissional dos professores foi, na visão dos comissários, muito teórico e desprovido de quaisquer inovações que estivessem sendo desenvolvidas no sistema educacional. Enfim, pensou-se que os professores de escolas normais não tinham oportunidade de desenvolver pesquisa em educação.

A reforma proposta pelo relatório Parent tinha por objetivo aumentar o nível da formação de professores para quebrar o isolamento das escolas universitárias de educação e para dar mais importância à pesquisa na sociedade franco-canadense. De fato, os comissários acreditavam que as universidades franco-canadenses não davam a devida importância à área de educação nem à pesquisa neste campo, geralmente desperdiçando seus esforços. Essas discussões levaram à criação, na metade dos anos de 1960, das primeiras faculdades de educação em três universidades de língua francesa - Universidade de Montreal, Universidade de Sherbrooke e Universidade Laval. Nesse contexto, depois de 15 anos de prevaricações, as escolas normais foram definitivamente abolidas em 1969 e todo o sistema de formação de professores ficou sob a responsabilidade das universidades.

Enquanto a ENS da cidade de Quebeque estava temporariamente em comutação, acompanhando a criação da Faculdade de Educação da Universidade Laval em 1965, a Escola de Ciências Pedagógicas e Psicológicas (o novo nome da EPO) foi integrada à faculdade. Como resultado dessa reorganização, o número de estudantes quadriplicou e o de professores dobrou. A EPO estava pronta para se tornar faculdade de educação, dedicada à formação de professores e de especialistas em educação e orientação vocacional, pois já concedia os graus de doutor e de mestre, bem como o de bacharel em educação, além de estar fortemente voltada para a cultura da pesquisa.

O fato de a ENS não estar incluída na Faculdade de Educação pode ser explicado, entre outras coisas, por suas ligações com as disciplinas de ensino. Dedicando-se principalmente ao treinamento de professores para se tornarem especialistas em um certo número de disciplinas para escolas secundárias e faculdades recém-fundadas (Faculdade de Ensino Geral e Profissional - CEGEPS), essa instituição manteve vínculos com as faculdades (de arte, ciências, etc.) e tentou preservar sua autonomia tanto quanto possível.

Por outro lado, por terem se mantido junto à Comissão Parent, as EPO reformistas militaram em favor da Faculdade de Educação e 
advogaram um modelo completamente diferente de formação de professores, em que a dimensão pesquisa deveria ser integrada com base em paradigmas que diferiam completamente dos modelos prévios de formação. Enquanto para a ENS a especialização visava principalmente a dialética entre disciplinas e treinamento pedagógico, para a EPO, a pesquisa desempenhava um papel mais importante do que a simples distribuição de diplomas de graduação. A EPO se concentrou em projetos de pesquisa financiados por organismos patrocinadores.

Mas como a cultura da pesquisa evoluiu nesta nova faculdade, que agora era responsável pela formação de professores primários e secundários? Uma rápida olhada nos indicadores dessa cultura da pesquisa mostra como a situação se desenvolveu, primeiro em relação à elaboração das diretrizes da instituição e, segundo, em relação aos professores.

No início, em 1965-1966, a faculdade foi organizada em torno de apenas dois departamentos: educação e orientação profissional. O primeiro visava a formação de professores, fornecendo-lhes cultura geral e prática. $\mathrm{O}$ segundo se concentrava na formação de especialistas, orientadores e cientistas de pesquisa. A faculdade concedia títulos de mestre e de doutor em educação e em orientação vocacional. Além disso, havia quatro opções na graduação incluindo uma especialização em pesquisa com enfoque em medidas e metodologia. Em 1967-1968, essa organização departamental binária mudou, pois a administração da faculdade julgou-a inadequada para o desenvolvimento de estudos de pós-graduação e de pesquisa. Equipes de pesquisa foram então organizadas e os professores agrupados, de acordo com o programa de pós-graduação. Entretanto, a partir de 1973, durante um período de grande crescimento no número de estudantes e de programas (1970 a 1980), a faculdade foi estruturada em torno de sete departamentos. Nesse período, a pesquisa se expandiu na maioria dos departamentos da faculdade graças ao apoio das bolsas obtidas pelos professores e também da criação e do desenvolvimento de laboratórios de pesquisa. ${ }^{11}$

Os fatores que contribuíram para o rápido desenvolvimento da faculdade foram: a necessidade, urgente e ampla, de treinamento na área da educação para a sociedade; a preocupação por parte dos professores da faculdade de exercer sua liderança na educação e desempenhar um papelchave na reforma educacional; e a extensão na área de educação. A faculdade queria manter as tensões criativas entre as tendências rumo à diferenciação e à integração. ${ }^{12} \mathrm{Em}$ suma, enquanto em 1965 não havia sequer um programa de mestrado e havia poucos estudantes no nível de

11 Ver Plano de três anos da Faculdade de Educação da Universidade Laval, 1979-1982.

12 Ver 25 aniversário da Faculdade de Educação da Universidade Laval. 
doutorado em orientação vocacional, em 1990 a Faculdade de Educação da Universidade Laval ofereceu dez programas de estudo em nível de mestrado e sete em nível de doutorado. A última reorganização, chamada "racionalização", foi realizada nos últimos dez anos, durante os quais a faculdade foi dividida em três departamentos ${ }^{13}$ com bons laboratórios, grupos de pesquisa e centros de pesquisa. A pesquisa tem, atualmente, um papel de destaque, especialmente se nos basearmos nos indicadores da evolução qualitativa e quantitativa dos professores.

Alguns dados nos ajudam a avaliar o surgimento da cultura da pesquisa na Faculdade de Educação da Universidade Laval. Em primeiro lugar, a composição do grupo de professores na faculdade mudou bastante, especialmente em comparação à sua instituição anterior, a EPO. A análise dos calendários da instituição mostrou que a proporção de mulhere ${ }^{14} \mathrm{e}$ professores leigos ${ }^{15}$ aumentou gradualmente, mas as mudanças principais estavam relacionadas à qualificação dos professores. A Faculdade de Educação progrediu muito neste aspecto se considerarmos que, em 1950, somente 39\% dos professores da EPO tinham o grau de doutor $(\mathrm{PhD})$.

Em 1996, 90\% dos professores da faculdade possuíam $\mathrm{PhD}$, enquanto em 1966 esse número era menor que $25 \%$. As principais razões para essa mudança foram: primeiro, o critério de contratação e de promoção de professores dentro do sistema hierárquico da universidade; segundo, o fato de que a universidade, por meio dos programas de bolsa e desenvolvimento profissional (do governo ou da universidade), motivou fortemente os professores a ampliarem suas qualificações. Esse esforço incansável alcançou seu pico nos anos 70: em 1976, 33\% dos professores da faculdade estavam cursando doutorado. Uma análise dos calendários da faculdade mostra que $60 \%$ deles obtiveram seu PhD desse modo.

Resumindo, baseados nos indicadores de qualificação de professores e na nossa consideração de que ter um $\mathrm{PhD}$ está diretamente relacionado ao desenvolvimento da pesquisa, podemos dizer que a cultura da pesquisa na Faculdade de Educação realmente decolou nos anos 70 .

13 O departamento de didática e de tecnologia psicoeducacional e educacional; o departamento de orientação profissional, administração e a avaliação; o departamento de educação física.

14 Em 1950, 8\% dos professores da EPO eram mulheres e 17\% em 1958. Nos anos de 1960, a proporção de mulheres na Faculdade de Educação era de apenas 11\%, aumentando para 27\% em 1996.

15 Em 1950, 41\% dos professores da EPO eram pessoas vindas de comunidades religiosas e $33 \%$ em 1958. Na Faculdade de Educação, essa característica baseada no status não figurava mais nos calendários. 
Descobrimos que as atividades de pesquisa são, agora, parte integrante da tarefa dos professores dentro da faculdade, enquanto na era da EPO somente uns poucos professores dedicavam seu tempo livre, ou no período de verão, à pesquisa. Em 1994-1995, dos 138 professores da Faculdade de Educação, 55 deles (40\%) estavam diretamente envolvidos, de um modo ou de outro, em atividades de pesquisa (Bureau du décanat, 1995).

Figura 2: Crescimento, em porcentagem, do grau de doutor (PhD) entre professores da Faculdade de Educação da Universidade Laval. Período de 1966 a 1996.

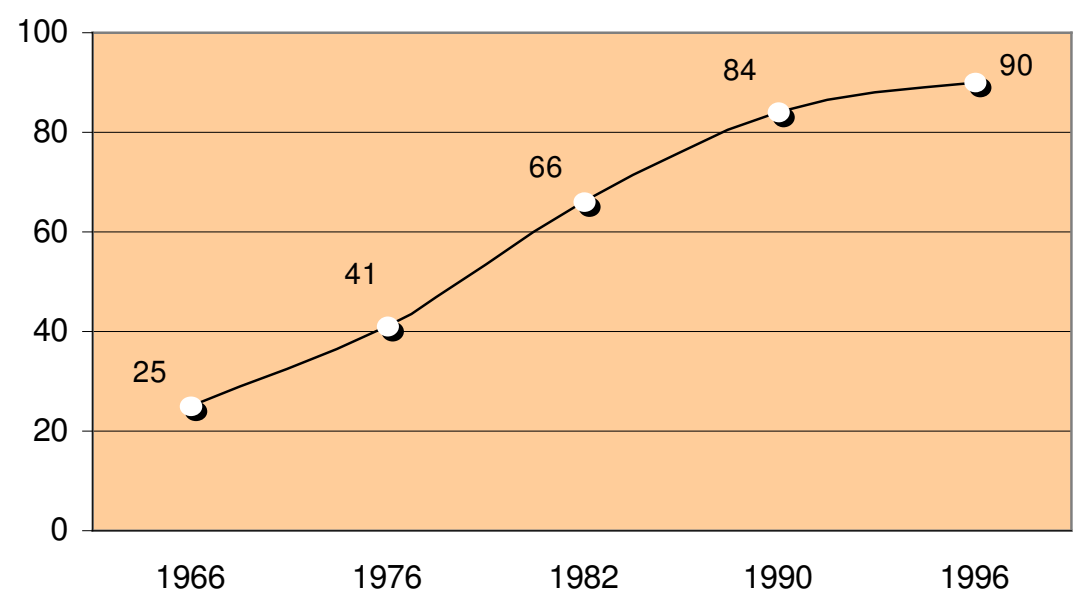

Fonte: Anais da Faculdade das Ciências da Educação, Universidade Laval.

Voltando nossa atenção para os aspectos qualitativos da trajetória de alguns professores, ou seja, suas tarefas, funções, atividades fora da universidade e suas qualificações, observamos que os professores da EPO possuíam múltiplas atividades. Por exemplo, Monsenhor Parent era secretário geral, vice-reitor e reitor da Universidade Laval além de Presidente da Comissão que tem seu nome. R. Miville, outro diretor da EPO, também acumulava títulos de alto nível, incluindo o de diretor da Faculdade de Artes. Alguns dos outros professores da instituição anterior eram médicos doutores, como, por exemplo J.-C. Miller, R.Blanchet e J.Gilbert. Os professores estavam freqüentemente ligados administrativamente a outras escolas, faculdades ou institutos (por exemplo, como chefes de clínicas psicológicas em hospitais, chefes de 
centros de reabilitação, como professores nas faculdades de filosofia, medicina, economia doméstica, escola de comércio ou de trabalho social, e assim por diante). Alguns envolviam-se em instituições pré-universitárias como a Académie de Québec, o Séminaire de Rimouski, o Collège Sainte-Anne de la Pocatière e o Collège Bourget. Entre as raras mulheres da EPO, uma era diretora de um centro de psicologia e outra diretora de uma instituição préescolar.

Como tinham outras atividades fora da Universidade Laval e da $\mathrm{EPO}$, podemos dizer que esses professores também estavam envolvidos com pesquisa em instituições públicas ou privadas. Por exemplo, o Arcebispo P. foi chefe da pesquisa educacional da Comissão das Escolas Católicas de Quebec (CECQ) de 1949 a 1951; Irmão Clément foi editor do jornal Pédagogie-orientation de 1952 a 1954; Dr. Gilbert foi diretor de educação no Ministério da Saúde e conselheiro no Conselho da Instrução Pública no subcomitê de saúde em 1947-1948. Além de exercer várias funções administrativas na universidade (recrutamento, treinamento externo, organização das aulas de verão), as múltiplas atividades dos professores da EPO mostram que, antes de 1960, além da colaboração entre escolas, faculdades e instituições também se envolviam com a sociedade e eram muito requisitados, tanto dentro quanto fora da universidade.

A análise qualitativa das trajetórias individuais mostra que o envolvimento com a pesquisa não era totalmente ausente, mas ocorria de diferentes formas, dependendo dos períodos considerados (publicação de livros religiosos, memórias, contribuições para várias autoridades, etc.). Assim, os pioneiros da EPO tinham vasta experiência devido a uma cultura geral ampliada, bem como múltiplas atividades e, ainda, uma grande dedicação social.

Em comparação, os professores da Faculdade de Educação estavam muito mais envolvidos com atividades diretamente relacionadas a seu trabalho profissional. Como a estrutura da universidade estava se desenvolvendo, e com ela uma burocracia cada vez mais freqüente e onerosa, os professores eram mais do que nunca requisitados para exercer numerosas tarefas administrativas: comissão de estudos, conselho universitário, secretaria de pós-graduação, vários comitês (de biblioteca, doutorados honorários, padrões de admissão, etc.) e por organizações tais como o sindicato de professores (de 1976 em diante). Fora da universidade, os professores continuam ocupados por muitas atividades profissionais, mas estas são diretamente relacionadas à sua posição e às suas responsabilidades na faculdade (associações de diretores ou de professores, etc.) Em suma, parece haver mais especialização na Faculdade de Educação do que na EPO, haja vista o que é revelado em calendários e em outras 
publicações. Seu intenso envolvimento social parece que foi substituído por atividades mais ligadas à profissão acadêmica. Nossa análise mostra que, comparando-a à EPO, surgiu na Faculdade de Educação uma cultura da pesquisa mais ativa, relacionada ao desenvolvimento da profissionalização e, em particular, da especialização.

\section{RUPTURAS NO SURGIMENTO DA CULTURA DA PESQUISA EM EDUCAÇÃO}

O fato de a Universidade Laval ter reconhecido, nos últimos 10 anos, três centros de pesquisa dentro da Faculdade de Educação, torna-se particularmente interessante discutir, no momento, as transições e períodos-chave no movimento em direção ao desenvolvimento da cultura da pesquisa em educação. Para manter a coerência, identificamos previamente a presença de uma cultura da pesquisa ou, ao contrário, uma cultura orientada para a formação de professores, ao considerar cada grupo de instituições independentemente. Agora ligaremos os períodos que estão relacionados com a cultura da pesquisa, na tentativa de entender como foram influenciados pelo desenvolvimento da universidade e da sociedade como um todo. Identificamos três períodos-chave:

Pré-1955 - uma cultura da pesquisa em formação

Antes de 1955, não havia praticamente nenhuma cultura da pesquisa, ou seja, nada nas escolas normais, nada ou muito pouco na ENS e somente uma cultura da pesquisa em formação na EPO. Grande parte da missão das três instituições era voltada ao preparo de professores para suas novas tarefas na sociedade. A ENS e a EPO já faziam parte de um movimento de "universitarização", mas somente a EPO se dedicava parcial e verdadeiramente à pesquisa.

De 1955 a 1969: os caminhos sinuosos em direção à "universitarização" da formação de professores

Para consolidar um sofisticado sistema de organização da pesquisa, nas instituições de formação de professores, essa rede de ensino primeiramente teve de passar a fazer parte da universidade. Mesmo antes que a formação de professores fosse oficialmente transferida para a universidade, em 1969, várias medidas foram tomadas para aumentar o nível da formação de professores nessas instituições e para aproximá-las 
das universidades. ${ }^{16} \mathrm{O}$ desenvolvimento das escolas universitárias de educação fez parte desta "longa marcha" em direção à universidade e um dos passos preliminares rumo à cultura da pesquisa. $\mathrm{Na}$ verdade, as universidades já estavam envolvidas nessa área antes da publicação do relatório Parent. Elas começaram a participar no mundo da pesquisa por meio de diferentes métodos, como, por exemplo, pela expansão do papel das escolas universitárias de educação. De modo similar, este período abriu caminho para a transferência oficial da formação de professores para a universidade e a EPO passou a dedicar-se inteiramente à pesquisa.

\section{De 1969 ao terceiro milênio: a cultura da pesquisa predomina}

De 1969 até a atualidade, e, particularmente, depois que a formação de professores foi oficialmente transferida para a universidade, parece que houve um movimento progressivo em direção à cultura da pesquisa. Primeiro, havia um forte desejo de contratar professores com $\mathrm{PhD}$ ou, quando a Faculdade de Educação contratava pessoal de escolas normais que tinham sido fechadas, de dar bolsas aos candidatos de modo que eles pudessem obter esse grau. Além disso, o desenvolvimento profissional dos professores, que foi induzido pela "Revolução Silenciosa", intensificou o desenvolvimento de estudos de pós-graduação em educação, já que muitos professores que estavam trabalhando entraram na universidade buscando o grau de PhD para melhorar seu treinamento. De acordo com Roby (1996), os esforços da universidade para desenvolver um pessoal de ensino portador de $\mathrm{PhD}$, incentivar estudos de pós-graduação e estimular a pesquisa partiram do reitor Kerwin no início da década de 1970. No mesmo ano, a Comissão de Pesquisa da Universidade Laval publicou um relatório (Comissão de pesquisa, 1971) que promoveu fortemente o desenvolvimento de grupos de pesquisa, incentivos para os professores se envolverem em centros interdisciplinares de pesquisa, incentivos para manter ou atrair excelentes pesquisadores e a reorganização de tarefas para que os professores tivessem mais tempo para desenvolver pesquisas. Do ponto de vista da universidade, esses fatores são, atualmente, parte integrante do programa de pesquisa.

A Faculdade de Educação, por sua vez, mudou no sentido de estimular a pesquisa, contratando somente professores com doutorado e

16 Por exemplo, o acordo de 1957 sobre a equivalência do diploma Classe A e um Bacharelado em Educação oferecido pelas escolas universitárias de educação, de acordo com a Lei para facilitar a formação do corpo docente em 1961. Essas duas medidas abriram as portas para que as pessoas que estudaram em escolas normais tivessem um nível mais elevado de educação. 
também pela organização de infra-estruturas direcionadas ao apoio e estímulo dos trabalhos de pesquisa dos professores. Os grupos de pesquisa, centros de pesquisa e laboratórios tornaram-se parte integrante do arsenal que toda a faculdade precisa possuir para ter sucesso na obtenção de bolsas. Na última década, houve grande enfoque na obrigatoriedade de que os membros da faculdade recém-contratados produzissem resultados. A pesquisa tornou-se parte importante da identidade dos professores da universidade no campo da educação.

\title{
CONCLUSÃO
}

A análise feita mostrou como a missão da universidade evoluiu através dos anos. Em seu livro, Jean Hamelin (1995) mostrou como, por longo tempo após sua criação, a Universidade Laval se concentrou, principalmente, na educação e na formação de cidadãos humanistas e "cultos". Além disso, como apontou, a pesquisa teve um pequeno papel no modo como a universidade a definiu na elaboração de suas diretrizes.

\begin{abstract}
"Os membros do Conselho Universitário viram a universidade como um local de educação, o lugar onde a herança é transmitida e os indivíduos que são intelectualmente mais brilhantes que outros podem satisfazer sua sede de conhecimento. Essa noção de universidade se sobrepõe às tradições das antigas instituições britânicas, mas provém da tradição prussiana na qual a universidade é concebida como uma comunidade de pesquisadores dedicados à busca da verdade e, assim, ao progresso da ciência (...)" (Hamelin, 1995, p. 38) [tradução]
\end{abstract}

Como nos movemos de "uma incubadora de cristãos, eruditos e patriotas", usando as palavras de Hamelin, para uma comunidade de professores-pesquisadores divididos entre dois mandatos essenciais, ensino e pesquisa, ambos necessários e dialeticamente ligados, mas atualmente difíceis de reconciliar? Para responder a essa pergunta, precisamos estudar em profundidade as conseqüências das reformas da instituição universitária acerca do desenvolvimento da cultura da pesquisa nas faculdades de educação.

A história da evolução da cultura da pesquisa nas instituições de formação de professores mostra que certos períodos, caracterizados como rupturas, resultaram de um certo número de tendências sobrepostas. Após uma melhora gradual no treinamento e na sua lenta profissionalização, um elo cada vez mais claro entre a formação de professores e a universidade emergiu em um curto período durante os anos de 1950. O rápido 
desaparecimento das escolas normais, a transformação das escolas normais estaduais e a crescente importância das escolas universitárias de educação na formação de professores, criou, gradualmente, mais espaço para a cultura da pesquisa se desenvolver e se estabelecer dentro da instituição. Somadas a esses elementos estruturais, algumas figuras-chave tiveram um papel importante nesse movimento de "universitarização" e legitimação. Entre elas estavam Monsenhor Parent e Arthur Tremblay, dois idealizadores importantes na reforma da década de 1960 e que foram personagens centrais na EPO. Suas funções nos permitem entender como essa instituição caminhou rapidamente rumo à cultura da pesquisa, baseada no modelo que unia o ensino à pesquisa dentro das universidades.

As faculdades de educação, por sua vez, voltaram-se definitivamente para a pesquisa. Esse movimento cresceu muito na década de 1970. Na verdade, embora as escolas normais superiores e a universidade estivessem intrinsecamente ligadas, parece que o fechamento das escolas normais e a transferência da formação de professores para a universidade forneceu um direção para a formação de professores baseada em novos paradigmas. Primeiro, a faculdade teve que adaptar seu critério de seleção ao grau de doutor, um critério que, como sabemos, se tornou o mínimo exigido para a contratação de professores, independentemente da faculdade à qual estavam vinculados. Contudo, antes da transferência, esse critério não era particularmente valorizado pelas instituições de formação de professores, exceto por alguns professores que trabalhavam em escolas universitárias de educação. Após esse primeiro movimento, a cultura da pesquisa tornou-se cada vez mais importante na medida em que os professores da Faculdade de Educação da Universidade Laval dedicavam mais tempo à pesquisa. Assim, a Faculdade de Educação seguiu o modelo da Faculdade de Artes, onde, conforme Roby (1996) demonstrou bem, os professores estavam desenvolvendo pesquisas e orientando muitos estudantes de pós-graduação. ${ }^{17}$ Essa mudança em direção à cultura da pesquisa pode ser explicada, pelo menos em parte, como uma busca pela legitimidade da área de educação, que agora fazia parte de uma instituição, a universidade, cuja tarefa inclui o desenvolvimento do conhecimento.

$\mathrm{O}$ que explica tal mudança na cultura institucional e também a transição da pesquisa voluntária à compulsória? Como essas tensões e tradições se encaixaram na experiência de professores e administradores

17 Ainda é muito cedo para dizer se a mudança para a pesquisa foi mais rápida nas faculdades de educação, se comparada a outras faculdades. Todavia, os poucos dados exploratórios apresentados sugerem que pode ser errado pensar que as faculdades de educação só se voltaram recentemente para a pesquisa. Esse ponto de vista é tendencioso, apressado e infundado. 
que viveram nesses períodos-chave? Como as pessoas lidaram com as tensões entre, pelo menos, duas culturas: uma centrada na pesquisa e outra em uma atividade para treinar profissionais de ensino? Estavam estas tensões ligadas de um modo criativo, como proposto pelos professores da faculdade nos últimos dez anos, ou provocaram uma tensão destrutiva, criando, assim, um conflito entre as prioridades de duas culturas contraditórias tentando se tornar predominantes ou hegemônicas? Isso é o que exploraremos por meio de entrevistas nos próximos estágios de nosso estudo sobre tradições e transições que, por meio de uma cultura da pesquisa, marcaram o desenvolvimento da educação e da formação de professores em Quebeque. Esperamos que a pesquisa nos ajude a preencher as lacunas do nosso conhecimento sobre as instituições universitárias, permitindo-nos, assim, conhecer melhor o papel desempenhado pela cultura da pesquisa na área de educação. Esse aspecto precisa ser melhor examinado e este estudo é uma tentativa de caminhar nessa direção. Estudos similares de outras áreas nos ajudarão a compreender as características, a continuidade e a ruptura entre a área de educação e outras faculdades. Também estaremos aptos para compreender como uma trajetória individual está interligada à história institucional, cultural e sociopolítica do seu ambiente. Finalmente, este estudo nos ajudará a entender as profundas e talvez inesperadas conseqüências de algumas tendências básicas dentro das universidades nos últimos 30 ou 40 anos. Quais são os modelos e paradigmas que informam as universidades e quais as conseqüências para os atores sociais que aí trabalham? Nossos projetos, que estão relacionados aos de equipes que trabalham em outros contextos nacionais, particularmente a Suíça, Islândia e Suécia, nos permitirão levantar pontos de vista convergentes e divergentes em uma perspectiva comparativa.

\section{REFERÊNCIAS BIBLIOGRÁFICAS}

ACKER, Sandra. Traditions and Transitions in Teacher Education: The Development of a Research Project, Historical Studies in Education/ Revue d'histoire de l'éducation, n.12, 1 \& 2 (spring/printemps \& Fall/automne 2000), p. 143-154. 
ALLARD, Michel (dir.) et al. Société canadienne pour l'étude de l'éducation. A Challenge met: The definition and recognition of the field of education/Un défi relevé. La définition et la reconnaissance du champ d'étude de l'éducation. Ottawa, Canadian Society for the Study of Education/Société canadienne pour l'étude de l'éducation (CSSE/SCEE), 1999, 209 p.

BUREAU DU DÉCANAT. Restructuration départementale. Rapport d'étape, Sainte-Foy, Québec, Faculté des sciences de l'éducation, Université Laval, $1995,57 \mathrm{p}$.

CALAM, John. Becoming a teacher: some historical perspectives. Alberta Journal of Educational Research, 27, 3, 1994, p. 272-284.

CHARTRAND, Luc; RAYMOND DUCHESNE, Michel Dubord; GINGRAS, Yves. Histoire des sciences au Québec. Montréal, Boréal, 1987, 487 p.

COMMISSION DE LA RECHERCHE. La réorganisation de la recherche à l'Université Laval. Sainte-Foy, Québec, Université Laval, 1971, 134 p.

CORBO, Claude. Matériaux fragmentaires pour une histoire de l'UQAM. De la descente aux enfers à l'UQAM de l'an 2000. Montréal, les Éditions Logiques, $1994,367 \mathrm{p}$.

FULLAN, Michael. Foreword. In: BOOTH, David; STIEGELBAUER, Suzanne (eds.). Teaching Teachers: the Faculty of Education University of Toronto 1906-1996, Hamilton, Ontario, 1996, i-xiv p.

HAMEL, Thérèse. Le déracinement des écoles normales. Le transfert de la formation des maîtres à l'université. Québec, Institut québécois de recherche sur la culture, 1991, $230 \mathrm{p}$.

Un siècle de formation des maîtres au Québec 1836-1939. Montréal, Hurtubise $\mathrm{HMH}, 1995,374$ p.

HAMELIN, Jean. Histoire de l'Université Laval: Les péripéties d'une idée, Sainte-Foy, Québec, Les Presses de l’Université Laval, 1995, 341 p.

MATHURIN, Creutzer. Les conditions sociohistoriques de la création des facultés de sciences de l'éducation au Québec. Thèse de doctorat, Faculté des études supérieures, Université de Montréal, 1992, 429 p. 
OPENSHAW, Roger. Forward to the Past in New Zealand Teacher Education. Journal of Education for Teaching, 25, 2, 1999, p. 111-122.

OPENSHAW, Roger. Some Historical Influences Upon Current Tertiary Mergers. Delta, 50, 1, 1998, p. 41-64.

PATERSON, Robert S. History of teacher education in Alberta. In: JONES, David C.; SHEEHAN, Nancy; STAMP, Robert (eds.). Shaping the Schools of the Canadian West, Calgary, Detselig, 1979, p. 192-207.

ROBY, Yves. Rapports enseignement recherche: équilibre rompu? In: ROBY, Yves; VOISINE, Nive (ed.). Érudition, humanisme et savoir. Actes du colloque en l'honneur de Jean Hamelin. Sainte-Foy, Québec, Les Presses de l'Université Laval, 1996, p. 29-49.

SHEEHAN, Nancy; WILSON, Donald J. From normal school to the university to the College of Teachers: teacher education in British Columbia in the $20^{\text {th }}$ century. Journal of Education for Teaching, 20, 1, 1994, p. 23-37.

Recebido em: outubro 2003 Aprovado para publicação em: setembro 2004 